\title{
PERFIL DE PESSOAS COM E SEM COMORBIDADES ACOMETIDAS POR REAÇÕES HANSÊNICAS*
}

Ana Paula Santos de Medeiros, Tatiane Aparecida Queiroz², Francisca Patrícia Barreto de Carvalho ${ }^{3}$, Clélia Albino Simpson ${ }^{4}$, Francisco Arnoldo Nunes de Miranda ${ }^{5}$, Eulália Maria Chaves Maia ${ }^{6}$

'Psicóloga. Mestranda em Psicologia. Universidade Federal do Rio Grande do Norte. Natal-RN-Brasil. ${ }^{2}$ Acadêmica de Enfermagem. Universidade do Estado do Rio Grande do Norte. Mossoró-RN-Brasil.

${ }^{3}$ Enfermeira. Doutoranda em Enfermagem. Professora da Universidade do Estado do Rio Grande do Norte. Natal-RN-Brasil. ${ }^{4}$ Enfermeira. Doutora em Enfermagem. Professora da Universidade do Estado do Rio Grande do Norte. Natal-RN-Brasil. ${ }^{5}$ Enfermeiro. Doutor em Enfermagem. Professor da Universidade do Estado do Rio Grande do Norte. Natal-RN-Brasil. ${ }^{6}$ Psicóloga. Doutora em Psicologia. Professora da Universidade do Estado do Rio Grande do Norte. Natal-RN-Brasil

RESUMO: Objetivou-se identificar características clínicas e epidemiológicas em pacientes com e sem comorbidades por hanseníase e reações hansênicas. Pesquisa quantitativa, descritiva e correlacional, realizada por 65 entrevistas em um centro de referência potiguar, com coleta de dados entre outubro de 2013 e março de 2014. Os homens representaram mais o grupo sem comorbidades, enquanto as mulheres e os idosos o grupo com comorbidades. Altas frequências nos dois grupos apontam para a forma Virchowiana como mais incidente e para algum grau de incapacidade. Houve correlação positiva entre quantidade de outros medicamentos versus outras comorbidades e negativa entre quantidade de pessoas que moram com o entrevistado versus número de comorbidades que este possui. Foram evidenciados diagnósticos tardios em virtude de altas frequências de incapacidades, bem como grupos de risco para reações hansênicas e comorbidades, composto por mulheres, idosos, pessoas com baixa escolaridade, que não trabalhavam e que utilizavam maior quantidade de medicamentos.

DESCRITORES: Hanseníase; Mycobacterium leprae; Doenças transmissíveis; Doenças negligenciadas.

\section{PROFILE OF PERSONS WITH AND WITHOUT COMORBIDITIES, STRICKEN BY LEPROSY REACTIONS}

\begin{abstract}
This study aimed to identify clinical and epidemiological characteristics in patients with and without comorbidities from leprosy and leprosy reactions. It is quantitative, descriptive and correlational research, undertaken through 65 interviews in a specialized center in Rio Grande do Norte, Brazil, with data collected between October 2013 and March 2014. The men represented most of the group without comorbidities, while women and older adults represented the group with comorbidities. High frequencies in the two groups point to the Lepromatous form as the most common, and to some degree of disability. There was positive correlation between number of other drugs versus other comorbidities and negative correlation between number of people who live with the interviewee versus the number of comorbidities which the interviewee has. Late diagnosis was evidenced due to high frequencies of disabilities, as well as groups at risk for leprosy reactions and comorbidities, made up of women, older adults, people with low educational level, people who did not work and those who used a higher number of medications. DESCRIPTORS: Leprosy; Mycobacterium leprae; Communicable diseases; Neglected diseases.
\end{abstract}

\section{PERFIL DE PERSONAS CONY SIN COMORBILIDADES ACOMETIDAS POR REACCIONES DE LA ENFERMEDAD DE HANSEN}

RESUMEN: Estudio cuyo propósito fue identificar características clínicas y epidemiológicas en pacientes con y sin comorbilidades por enfermedad de Hansen y reacciones a esa enfermedad. Investigación cuantitativa, descriptiva y correlacional, realizada por 65 entrevistas en un centro de referencia. Los datos fueron obtenidos entre octubre de 2013 y marzo de 2014. Había más hombres en grupo sin comorbilidades, en cuanto las mujeres y los ancianos representaron más el grupo con comorbilidades. Altas frecuencias en los dos grupos apuntan para la forma Virchowiana como la con más incidencia y para algun grado de incapacidad. Hubo correlación positiva entre cantidad de otros medicamentos versus otras comorbilidades y negativa entre cantidad de personas que viven con el entrevistado versus número de comorbilidades que este presenta. Fueron evidenciados diagnósticos tardíos en función de altas frecuencias de incapacidades, así como grupos de riesgo para reacciones hansénicas y comorbilidades, compuesto por mujeres, ancianos, personas con poca escolaridad, que no trabajaban y que utilizaban mayor cantidad de medicamentos. DESCRIPTORES: EnfermedaddeHansen; Mycobacterium leprae; Enfermedades transmisibles; Enfermedades negligenciadas.

\footnotetext{
*Artigo extraído da monografia de conclusão de curso de gradução em Enfermagem, intitulada: Características Clínicas e Epidemiológicas de Indivíduos em Reação Hansênica Atendidos em um Centro de Referência para a Hanseníase no Município de Mossoró-RN. Universidade do Estado do Rio Grande do Norte, 2015.
}

\section{Autor Correspondente:}

Ana Paula Santos de Medeiros/UFRN

Universidade Federal do Rio Grande do Norte

Rua Rio Guaíba, 7698 - 59068-260 - Natal-RN-Brasil

E-mail: apsm85_psi@yahoo.com.br
Recebido: 07/04/2015

Finalizado: 15/05/2015 


\section{INTRODUÇÃO}

A hanseníase é uma das doenças mais antigas que acometem o homem. Suas referências datam de 600 a. ${ }^{(1)}$ e desde os tempos mais remotos tem sido considerada contagiosa e mutilante, trazendo rejeição, discriminação e exclusão do doente na sociedade(2).

É uma doença infecto-parasitária, crônica, granulomatosa e de evolução lenta, causada pelo Mycobacterium leprae, bacilo que infecta predominantementeas células cutânease dos nervos periféricos, ocasionando lesões nesses locais ${ }^{(3)}$.

As principais causas de morbidade da hanseníase estão relacionadas às deformidades e incapacidades físicas. Esses agravos tem origem nas reações hansênicas ou estados reacionais, que são ocasionados por reações do sistema imunológico da pessoa atingida pela hanseníase ao bacilo causador da doença. As reações são classificadas em tipo I, também conhecida como reação reversa ou em tipo II, também denominada de eritema nodoso hansênico ${ }^{(1)}$.

As reações hansênicas se constituem como um importante evento na evolução da hanseníase, tendo em vista que não há um tratamento específico que possa impedir sua ocorrência, não há esquemas de tratamento eficazes para todos os casos e que durante os episódios reacionais ocorre a piora das lesões neurológicas e consequentemente o aumento das incapacidades físicas ${ }^{(4)}$.

O preconceito e a diminuição da qualidade de vida também se acentuam com a presença dos estados reacionais, que interferem diretamente nas condições físicas, psicológicas, sociais e econômicas de seus portadores, que passam a sentir vergonha de si mesmo, a sofrer com o desemprego, além de enfrentarem problemas no casamento e dificuldades em encontrar parceiros $^{(5)}$.

Além disso, as complicações provocadas pela hanseníase vêm suscitando mais a cada dia a necessidade de atenção, seja em decorrência de suas próprias peculiaridades, seja pelas associações da doença a outros agravos ${ }^{(6)}$. Aliás, a própria reação hansênica é apontada como sendo o motivo mais frequente de óbitos confirmados dessa patologia ${ }^{(7)}$.

Diante do que foi anteriormente discutido, entende-se que o atendimento aos indivíduos em estados reacionais deve ultrapassar a atenção voltada estritamente às queixas, sinais e sintomas trazidos pela hanseníase e/ou pelos estados reacionais, sendo necessário dar resposta às demais necessidades de saúde que os indivíduos apresentam, assim como as suas necessidades sociais, econômicas ou psicológicas.

Mediante os novos desafios sociais, políticos e culturais, o esgotamento do paradigma biomédico e a mudança do perfil epidemiológico da população, ocorrido nas últimas décadas, emerge a necessidade de uma mudança nas práticas assistenciais no sentido de conceber o indivíduo enquanto sujeito, inserido em diferentes contextos e influenciado diretamente pelos determinantes sociais, ou seja, compreendê-lo em sua integralidade ${ }^{(8)}$. O objetivo deste estudo foi identificar as principais características clínicas e epidemiológicas em pacientes com e sem comorbidades acometidos por hanseníase e reações hansênicas.

\section{MÉTODO}

Para alcançar o objetivo proposto foi realizada uma pesquisa de abordagem quantitativa, de natureza descritiva e correlacional e de corte transversal ${ }^{(9)}$. A amostra que resultou em 65 participantes foi calculada conforme fórmula estatística. Foram incluídos os pacientes que realizavam acompanhamento periódico para o tratamento de reações hansênicas no centro clínico de referência escolhido para o estudo, sendo eles de qualquer idade ou sexo. As pessoas com reações hansênicas que não faziam tratamento no centro clínico, bem como as consideradas curadas, que somente faziam tratamento para doenças acarretadas pela hanseníase foram excluídas do estudo. Após conhecida a amostra, esta passou a ser dividida em dois grupos, o primeiro composto por pacientes com hanseníase e reações hansênicas $(N=37)$ e o segundo com hanseníase, reações hansênicas e comorbidades ( $N=28$ ).

O cenário de pesquisa escolhido foi um centro clínico de referência para tratamento da hanseníase da cidade de Mossoró e da mesorregião do Oeste Potiguar. Trata-se de uma unidade de atendimento multiprofissional 
composta por sete profissões que realizam todas as atividades propostas do Programa de Controle da Hanseníase.

Para a coleta de dados realizou-se entre os meses de outubro de 2013 e março de 2014 uma entrevista semi-estruturada e um exame físico, realizados nesta ordem, individualmente, em local reservado e por meio de demanda espontânea. A entrevista foi composta por questões acerca de aspectos clínicos, sociais e epidemiológicos e o exame físico foi baseado em um roteiro norteador, construído a partir de instrumentos validados pelo Ministério da Saúde (Ficha-A do Sistema de Atenção Básica e o Prontuário para Acompanhamento de Casos de Hanseníase).

Os dados foram tabulados e analisados com o auxílio do software SPSS (Statistical package for the social sciences), versão 20.0. Foi utilizada a estatística descritiva, retornando frequências, médias e desvios padrões, e posteriormente utilizado o teste Qui-quadrado e a Correlação de Spearman, considerando para ambos o nível de significância de $5 \%(0,05)$. O objetivo dos testes foi comparar os resultados entre os grupos.

Foram levados em consideração os princípios éticos sugeridos para o caso, tais como aprovação do projeto pelo Comitê de Ética em Pesquisa sob parecer $n^{\circ}$ 356.765, respeito à Declaração de Helsink e à Resolução do Conselho Nacional de Saúde n.466, de 12/12/12. Foram adotados cuidados como solicitação de autorização de permissão para pesquisa por parte da instituição, privacidade na coleta e no armazenamento dos dados, fornecimento de informações gerais sobre a pesquisa e de condição voluntária de participação através do Termo de Consentimento Livre e Esclarecido, bem como respeito às condições gerais da pessoa humana.

\section{RESULTADOS}

O grupo formado por pacientes com comorbidades foi caracterizado por pessoas com até seis outras patologias, sendo referida na maioria das vezes a existência de uma delas $(60,7 \%)$. No total, foram citadas 36 diferentes patologias, sendo diabetes mellitus, hipertensão arterial e osteoporose/osteopenia igualmente mais frequentes, citadas seis vezes $(16,7 \%)$ cada uma. Os transtornos mentais foram referidos cinco vezes (13,9\%). Anemia, doenças renais, artrose e cardiopatia apareceram duas vezes cada e cinco outras patologias foram mencionadas apenas uma vez $(2,8 \%)$.

A análise dos resultados por comparação de grupos com e sem comorbidades sugere padrões diferenciados, entretanto, o teste Qui-Quadrado aponta diferenças significativas somente em termos de sexo $(p=0,046)$ e faixa etária $(p=0,035)$. Quanto ao sexo, a amostra é mais representativa de homens sem comorbidades (38,46\%); ao contrário, as mulheres foram mais frequentes no grupo com comorbidades (24,61\%). (Tabela 1$)$.

As idades variaram entre 13 e 79 anos $(M=$ 48,8; o = 0,499) e foram categorizadas em adultos e idosos (a OMS define a pessoa idosa como aquela com 60 anos ou mais). Como a idade de 13 anos foi apresentada por apenas um indivíduo, este foi incluído na categoria mais próxima, a dos adultos. Os adultos sem comorbidades representaram quase metade da amostra (49,2\%). Os idosos (15,4\%) estiveram mais presentes na categoria com outras comorbidades.

A maioria das pessoas foi proveniente da zona urbana de Mossoró (84,6\%) e, destas, $46,2 \%$ não referiram possuir outra patologia. Quando solicitadas a declarar sua cor, os menores percentuais foram encontrados na auto referência à cor parda, apenas 10,8\%. Vale ressaltar que a cor branca foi mais representada pelo grupo sem comorbidades $(33,8 \%)$ e a cor negra pelo grupo com comorbidades $(21,5 \%)$.

Das 24 ocupações citadas, do lar (13,8\%) foi a mais expressiva, seguida por aposentado (12,3\%), doméstica (10,7\%) e agricultor (9,2\%). Apenas duas pessoas relataram estar desempregadas. Onze ocupações apareceram apenas uma vez. Para facilitar a análise, as ocupações foram agrupadas em duas categorias (trabalhavam e não trabalhavam). A maior parte das pessoas que trabalhavam não possuíam comorbidades (44,6\%) enquanto a categoria formada por pessoas que não trabalhavam compôs-se em maior frequência por pessoas com comorbidades (18,4\%). Quando investigada a escolaridade, observa-se que a maioria (83\%) era analfabeta ou possuía o ensino fundamental incompleto. A renda familiar de ambos os grupos distribuem-se em maior parte na classificação de até um salário mínimo. 
De modo geral, a maioria das pessoas com e sem comorbidades responderam morar em casa própria (80\%), com água encanada (96,9\%), rede de esgoto ou fossa séptica (95,3\%), ter seu lixo coletado (93,8\%), energia elétrica (98,9\%). Os grupos não apresentaram grandes variações, no máximo duas pessoas em cada grupo não possuíam essas condições de moradia. Foram referidos até oito moradores em cada moradia, que possuíam até oito cômodos.

A análise correlacional por meio do teste de Rô de Spearman aponta para uma correlação negativa fraca $(o=-0,238 ; p=0,056)$, não significativa, entre quantidade de pessoas que moram com o entrevistado e número de comorbidades que possui. Isto é, quanto maior o número de pessoas morando com o entrevistando menor a quantidade de comorbidades identificadas, e o oposto também.

A Tabela 2 indica que 70,8\% dos pacientes foram diagnosticados com alguma incapacidade ao exame clínico, grau I ou II, com altas frequências nos grupos com e sem comorbidades. Ao investigar as formas clínicas da hanseníase, observa-se predomínio para as formas Virchowiana e Dimorfa, com maior frequência para a forma Virchowiana no grupo sem comorbidades, o que correspondeu a $32,3 \%$ do total. A forma Indeterminada não foi encontrada. (Tabela 2).

Tabela 1 - Pacientes com reações hansênicas em grupos com e sem comorbidades, segundo variáveis sociodemográficas. Mossoró-RN-Brasil, 2013-2014

\begin{tabular}{|c|c|c|c|c|c|c|}
\hline \multirow{3}{*}{ Variáveis } & \multicolumn{6}{|c|}{ Comorbidades } \\
\hline & \multicolumn{2}{|c|}{ Presentes } & \multicolumn{2}{|c|}{ Ausentes } & \multicolumn{2}{|c|}{ Total } \\
\hline & $\mathbf{n}$ & $\%$ & $n$ & $\%$ & $n$ & $\%$ \\
\hline \multicolumn{7}{|l|}{ Sexo } \\
\hline Feminino & 16 & $24,6 \%$ & 12 & $18,5 \%$ & 28 & $43,1 \%$ \\
\hline Masculino & 12 & $18,5 \%$ & 25 & $38,5 \%$ & 37 & $56,9 \%$ \\
\hline \multicolumn{7}{|l|}{ Faixa etária } \\
\hline Adultos & 18 & $27,7 \%$ & 32 & $49,2 \%$ & 50 & $76,9 \%$ \\
\hline Idosos & 10 & $15,4 \%$ & 5 & $7,7 \%$ & 15 & $23,1 \%$ \\
\hline \multicolumn{7}{|l|}{ Moradia } \\
\hline Zona Rural & 3 & $4,6 \%$ & 7 & $10,8 \%$ & 10 & $15,4 \%$ \\
\hline Zona Urbana & 25 & $38,5 \%$ & 30 & $46,2 \%$ & 55 & $84,6 \%$ \\
\hline \multicolumn{7}{|l|}{ Cor } \\
\hline Branca & 14 & $21,5 \%$ & 22 & $33,8 \%$ & 36 & $55,4 \%$ \\
\hline Negra & 14 & $21,5 \%$ & 8 & $12,3 \%$ & 22 & $33,8 \%$ \\
\hline Parda & 0 & $0,0 \%$ & 7 & $10,8 \%$ & 7 & $10,8 \%$ \\
\hline \multicolumn{7}{|l|}{ Ocupação } \\
\hline Não trabalha & 12 & $18,5 \%$ & 8 & $12,3 \%$ & 20 & $30,8 \%$ \\
\hline Trabalha & 16 & $24,6 \%$ & 29 & $44,6 \%$ & 45 & $69,2 \%$ \\
\hline \multicolumn{7}{|l|}{ Escolaridade } \\
\hline Analfabeto/Ensino Fundamental & 24 & $36,9 \%$ & 30 & $46,2 \%$ & 54 & $83 \%$ \\
\hline Ensino Médio & 4 & $6,2 \%$ & 7 & $10,7 \%$ & 11 & $16,9 \%$ \\
\hline
\end{tabular}

Questionados sobre o medicamento usado para o tratamento dos estados reacionais, chama atenção para um maior número de uso de prednisona associada à talidomida e a ausência do uso isolado da talidomida em pessoas com comorbidades. Sobre o uso de outros medicamentos, os entrevistados relataram fazer uso de até oito. Encontrou-se uma correlação positiva significativa $(o=0,257 ; p=0,039)$ entre quantidade de medicamentos e de outras comorbidades, indicadas pela correlação de Rô de Spearman. Apesar de fraca, indica que quanto maior o número de medicamentos utilizados pelos pacientes, maior também o número de comorbidades apresentadas por eles. 
Tabela 2 - Pacientes com hanseníase e reações hansênicas em grupos com e sem comorbidades, segundo grau de incapacidade atual e forma clínica. Mossoró, Rio Grande do Norte, Brasil, 2013-2014

\begin{tabular}{|c|c|c|c|c|c|c|}
\hline \multirow{3}{*}{ Variáveis } & \multicolumn{6}{|c|}{ Comorbidades } \\
\hline & \multicolumn{2}{|c|}{ Presentes } & \multicolumn{2}{|c|}{ Ausentes } & \multicolumn{2}{|c|}{ Total } \\
\hline & $\mathbf{n}$ & $\%$ & $\mathbf{n}$ & $\%$ & $n$ & $\%$ \\
\hline \multicolumn{7}{|l|}{ Sexo } \\
\hline Grau 0 & 9 & $13,8 \%$ & 10 & $15,4 \%$ & 19 & $29,2 \%$ \\
\hline Grau I & 10 & $15,4 \%$ & 18 & $27,7 \%$ & 28 & $43,1 \%$ \\
\hline Grau II & 9 & $13,8 \%$ & 9 & $13,8 \%$ & 18 & $27,7 \%$ \\
\hline \multicolumn{7}{|l|}{ Forma Clínica } \\
\hline Tuberculóide & 3 & $4,6 \%$ & 3 & $4,6 \%$ & 6 & $9,2 \%$ \\
\hline Dimorfa & 10 & $15,3 \%$ & 11 & $16,9 \%$ & 21 & $32,3 \%$ \\
\hline Virchowiana & 14 & $21,5 \%$ & 21 & $32,3 \%$ & 35 & $53,8 \%$ \\
\hline Neural Pura & 1 & $1,5 \%$ & 2 & $3,1 \%$ & 3 & $4,6 \%$ \\
\hline
\end{tabular}

\section{DISCUSSÃO}

Embora a hanseníase acometa ambos os sexos, também se observam em outros estudos a sua predominância entre os homens ${ }^{(1,10-11)}$. Entretanto, quando observado os indicadores de saúde em homens e mulheres do Brasil, percebese que as mulheres possuem menor desempenho geral em fatores como dieta, atividade física, peso corporal, colesterol alto, glicemia em jejum e pressão alta, por exemplo ${ }^{(12)}$, situação que contribui para o aparecimento de doenças. Isto indica que apesar dos programas e campanhas sugeridos pelo Ministério da Saúde do Brasil e voltados ao acompanhamento de patologias mais incidentes como hipertensão e diabetes, a promoção e prevenção da saúde não tem atingido um resultado satisfatório para as usuárias.

Existem relatos na literatura de longos períodos de incubação da hanseníase, chegando a 10 anos, motivo que aumenta a possibilidade de uma pessoa adoecer na fase adulta e idosa. A incidência de casos em menores de 15 anos é mais frequente em regiões endêmicas, como Mossoró, especialmente em focos domiciliares não tratados que expõem precocemente as crianças $^{(1)}$. Destaca-se a necessidade de atenção aos idosos por parte do sistema de saúde, visto sua maioria apresentar outras patologias além da hanseníase. Trata-se de um público já considerado mais exposto a patologias em geral especialmente se considerados os fatores biológicos, psicológicos e sociais da sua fragilidade ${ }^{(13)}$.
Os resultados mostram a cor da pele negra como mais associada a comorbidades. De fato, um estudo realizado no Brasil mostra que a maioria dos óbitos investigados em decorrência de complicações da hanseníase tem como perfil pessoas da cor preta ou parda ${ }^{(7)}$.

É importante ressaltar o possível incremento do apoio social junto ao número de pessoas que moram com o entrevistado, provocando uma correlação negativa com outras comorbidades. Este conceito envolve informação de qualquer origem, assistência material e proteção ofertadas por aqueles que propiciam contatos sistemáticos e efeitos positivos ${ }^{(14)}$. Portanto, pessoas que compartilham de relações sociais podem perceber-se como cuidado e amado, bem como pertencente a uma rede composta por figuras de apoio social. Esta condição se configura como proteção que constantemente resguarda o indivíduo de riscos ocasionados por crises patológicas ${ }^{(15)}$.

Em geral, características sociodemográficas encontradas nesse estudo convergem com as de outros realizados no Brasil que apontam as precárias condições socioeconômicas e informacionais como fatores que ajudam a manter altos índices de hanseníase ${ }^{(16-17)}$. A baixa escolaridade acarreta menor oportunidade de contato com informações coerentes e confiáveis sobre a doença e de trabalho com melhores remunerações, o que em conjunto pode influenciar diretamente em condições desfavoráveis de habitação, higiene e saúde dessas pessoas, promovendo condições ambientais para 
a infecção pelo M. leprae.

Altos índices de incapacidade em grupo com e sem comorbidades preocupa. A porcentagem encontrada neste estudo aparece alta $(70,8 \%)$ em comparação com um estudo realizado no Piauí, também da Região Nordeste do Brasil, onde aponta para $20 \%$ de casos da hanseníase resultante em algum grau de incapacidade ${ }^{(18)}$ e indica que os diagnósticos podem estar ocorrendo tardiamente. Além disso, os altos índices de incapacidade e a maior frequência de pessoas com comorbidades no grupo que não trabalhava parecem se justificar.

As incapacidades físicas podem ocasionar prejuízos que se estendem desde aos econômicos e sociais, quanto aos físicos e psicológicos. Mas as condições de saúde e de deficiência não parecem ser suficientes para explicar o desempenho ocupacional de pessoas com sequelas neurológicas ${ }^{(19)}$. Muitas vezes os fatores ambientais e pessoais como motivação e pobreza podem interferir no processo de busca pelo trabalho.

Quanto ao uso dos medicamentos para as reações hansênicas, sabe-se que o tratamento combinado com talidomida e prednisona é indicado para os casos cujas reações tipo 2 são mais intensas e de difícil controle. Esse tipo de reação também pode ser chamado de Eritema Nodoso Hansênico e é mediada por anticorpos (imunidade humoral) $)^{(1)}$. A talidomida é a droga de escolha para as reações tipo 2, porém esta não é indicada para mulheres em idade fértil devido aos seus efeitos teratogênicos. Como a amostra com comorbidades foi representada em grande parte por mulheres, entende-se uma quantidade inferior do uso isolado desse medicamento. Ainda assim, a maior frequência do uso combinado no grupo com comorbidades pode representar um grande risco em situação de gravidez ${ }^{(4)}$.

Vale salientar que apesar de seu importante efeito anti-inflamatório, o uso prolongado de corticosteroides pode provocar sérios efeitos adversos, tornando-se então um fator complicador da condição clínica do paciente, entre os efeitos adversos pode-se citar: alterações metabólicas como a intolerância a glicose e desenvolvimento ou descompensação do diabetes, elevação do colesterol e triglicerídeos e aumento da gordura intra-abdominal; alterações endócrinas como déficit de crescimento, alterações menstruais e pancreatite; alterações no balanço hidroeletrolítico do cálcio, potássio e sódio; alterações musculoesqueléticas como osteoporose, fraqueza muscular e perda de massa muscular; alterações hematológicas como leucocitose, diminuição de eosinófilos e de linfócitos e aumento de plaquetas e alterações renais como urolitíase ${ }^{(20)}$.

Além disso, podem ocorrer alterações gastrointestinais como esofagite, predisposição a úlceras gastroduodenais e distensão abdominal; alterações oftalmológicas como catarata e glaucoma; alterações sistema nervoso como hipertensão intracraniana, distúrbios do humor, psicoses, alterações da personalidade, convulsões, vertigem, cefaleia, irritabilidade e insônia; alterações cardiovasculares como hipertensão arterial sistêmica e desencadeamento ou agravamento de insuficiência cardíaca congestiva e alterações imunológicas contribuindo para o desenvolvimento de infecções e parasitoses ${ }^{(20)}$. Em decorrência desses inúmeros efeitos adversos, a maior ocorrência de comorbidades nos pacientes que fazem uso de prednisona pode estar diretamente relacionada à utilização prolongada desse medicamento.

Embora os pacientes utilizem vários outros medicamentos, além daqueles para o tratamento convencional das reações hansênicas, percebese que a maioria deles direcionam-se para os efeitos colaterais do tratamento ou implicações da hanseníase e pouco tem se investido em cuidados que buscam o controle das comorbidades. Portanto, apesar dos pacientes possuírem conhecimento sobre a presença de outras patologias, o tratamento medicamentoso parece insuficiente, o que reforça a necessidade de melhor acompanhamento e encaminhamento por parte dos profissionais que atuam com pessoas com reações hansênicas e comorbidades.

\section{CONCLUSÃO}

Ao cumprir o objetivo proposto no estudo, observou-se que uma das parcelas da população historicamente situada à margem da sociedade e constantemente alvo de preconceito apareceu acometida por hanseníase, reações hansênicas e comorbidades. Esta parcela foi composta especialmente por pessoas do sexo feminino, idosas, de baixa renda e escolaridade, e com 
algum grau de incapacidade, no qual obtiveram altas frequências na combinação dos três fatores de risco para a saúde. Destaca-se o risco da grande quantidade de medicamentos utilizados entre pessoas com reações hansênicas e comorbidades, sendo estes fármacos ainda não específicos ao tratamento das comorbidades; destaca-se também o benefício do maior número de outros moradores na residência da pessoa com hanseníase, relacionada à menor possibilidade que este tem de apresentar comorbidades.

$\mathrm{O}$ alto percentual de participantes com comorbidades reflete a necessidade de um acompanhamento integral de saúde voltado não somente ao acompanhamento de uma enfermidade ou medicalização dos sintomas, mas à busca de terapias especializadas objetivando antecipar diagnósticos, melhorar a qualidade de vida e modificar o atual perfil da hanseníase no Brasil. Ademais, é necessário aprimorar o trabalho de educação, supervisão, sensibilização e conscientização dos profissionais e das pessoas acometidas pela hanseníase a fim de antecipar diagnósticos, minimizar as incapacidades e melhorar a qualidade de vida destas.

As contribuições desse estudo estão em chamar a atenção para uma doença negligenciada e ainda esquecida pelos baixos investimentos em ações de controle e detecção, mas endêmica em muitas cidades como na investigada no presente estudo. A busca pela identificação de características clínicas e epidemiológicas em pessoas afetadas pela hanseníase e por comorbidades se torna mais uma ferramenta para o combate à doença, observada a dificuldade de identificar esse perfil e favorece o desenvolvimento de medidas preventivas que permitam a redução das incapacidades decorrentes das reações hansênicas.

Sabe-se que as propriedades das doenças variam não apenas de suas características intrínsecas, mas dependem, sobretudo, da relação individual do hospedeiro, fatores ambientais e da própria bactéria ${ }^{(1)}$. Portanto, recomendase o aprofundamento da temática em diversas localidades, investigando outras variáveis e com amostra ampliada, a fim de se conhecer melhor esse grupo de pessoas que lutam em exaustiva e duradora batalha, mas que convivem com variados sintomas e patologias que ameaçam sua saúde física, mental, econômica e social.

\section{REFERÊNCIAS}

1. Ministério da Saúde (BR). Guia de Vigilância Epidemiológica. Série A. Normas e Manuais Técnicos. $7^{\text {a }}$ Ed. Brasília: Ministério da Saúde; 2009.

2. Nunes JM, Oliveira EN, Vieira NFC. Hanseníase: conhecimentos e mudanças na vida das pessoas acometidas. Ciênc. saúde colet. 2011;16(1):1311-18.

3. Ministério da Saúde (BR). Guia de procedimentos técnicos: baciloscopia em hanseníase. Brasília: Ministério da Saúde; 2010.

4. Ura S. Tratamento e controle das reações hansênicas. Hansen. int. 2007;32(1):67-70.

5. Van Brakel WH, Sihombing B, Djarir H, Beise K, Kusumawardhani $L$, Yulihane $R$, et al. Disability in people affected by leprosy: the role of impairment, activity, social participation, stigma and discrimination. Glob. Health Action. [Internet] 2012;(5) [acesso em 10 out 2014]. Disponível: https://dx.doi.org/10.3402/gha. v5i0.18394.

6. Gonçalves A. Realidades do controle da hanseníase: atualizando cenários. Rev. Bras. Epidemiol. 2013;16(3):611-21.

7. Rocha MCN, Garcia LP. Investigação epidemiológica dos óbitos notificados tendo como causa básica a hanseníase, ocorridos em Fortaleza, Ceará, 2006-2011. Epidemiol. serv. saúde. [Internet] 2014;23(2) [acesso em 10 out 2014]. Disponível: http://dx.doi.org/10.5123/ S1679-49742014000200009.

8. Montenegro LC, Penna CMM, Brito MJM. A integralidade sob a ótica dos profissionais dos serviços de saúde de Belo Horizonte. Rev. Esc. Enferm. USP. 2010;44(3):649-56.

9. Gil AC. Métodos e Técnicas de Pesquisa social. $6^{\mathrm{a}}$ ed. São Paulo: Atlas; 2008.

10. Sousa MWG, Silva DC, Carneiro LR, Almino MLBF, Costa ALF. Epidemiological profile of leprosy in the Brazilian state of Piauí between 2003 and 2008. An. Bras. Dermatol. [Internet] 2012;87(3) [acesso em 16 out 2014]. Disponível: http://dx.doi.org/10.1590/S036505962012000300006.

11. Corrêa RGCF, Aquino DMC, Caldas AJM, Amaral DKCR, França FS, Mesquita ERRBPL. Epidemiological, clinical, and operational aspects of leprosy patients assisted at a referral service in the state of Maranhão, Brazil. Rev. Soc. Bras. Med. Trop. [Internet] 2012;45(1) [acesso em 16 out 2014]. Disponível: http://dx.doi. org/10.1590/S0037-86822012000100017.

12. Felisbino-Mendes MS, Jansen AK, Gomes CS, Velásquez-Meléndez G. Avaliação dos fatores de 
risco cardiovasculares em uma população rural brasileira. Cad. saude publica. [Internet] 2014;30(6) [acesso em 16 out 2014]. Disponível: http://dx.doi. org/10.1590/0102-311X00103213

13. Bandeira IC. Fragilidade em idosos: uma revisão integrativa [monografia]. Porto Alegre (RS): Universidade Federal do Rio Grande do Sul. [Internet] 2010 [acesso em 10 fev 2015]. Disponível: http://www. lume.ufrgs.br/handle/10183/28050?show=full.

14. Gonçalves TR, Pawlowski J, Bandeira DR, Piccinini CA. Avaliação de apoio social em estudos brasileiros: aspectos conceituais e instrumentos. Ciênc. saúde colet. [Internet] 2011;16(3) [acesso em 10 nov 2013]. Disponível: http://dx.doi.org/10.1590/S141381232011000300012.

15. Cobb S. Social support as a moderator of life stress. Psychosom. Med. 1976;38(5):300-14.

16. Aquino DMC, Caldas AJM, Silva AAM, Costa JML. Perfil dos pacientes com hanseníase em área hiperendêmica da Amazônia, Maranhão - Brasil. Rev. Soc. Bras. Med. Trop. [Internet] 2003;36(1) [acesso em 10 set 2014]. Disponível: http://dx.doi.org/10.1590/ S0037-86822003000100009.

17. Martins MA. Qualidade de vida em portadores de hanseníase [dissertação]. Campo Grande (MS): Universidade Católica Dom Bosco; 2009.

18. Lustosa AA, Nogueira LT, Pedrosa JIS, Teles JBM, Campelo V. The impact of leprosy on health-related quality of life. Rev. Soc. Bras. Med. Trop. [Internet] 2011;44(5) [acesso em 18 out 2014]. Disponível: http:// dx.doi.org/10.1590/S0037-86822011000500019.

19. Navarro EJ, Stoffel DP, Nickel R. A independência funcional e a manutenção dos papéis ocupacionais em sujeitos com sequelas neurológicas. Cogitare enferm. [Internet] 2013;18(4) [acesso em 03 jan 2015]. Disponível: http://dx.doi.org/10.5380/ce.v18i4.34919.

20. Ministério da Saúde (BS). Capacitação em prevenção de incapacidades em hanseníase: caderno do monitor. Brasília: Ministério da Saúde; 2010. 